\title{
Seroprevalence of Bordetella pertussis antibodies in adults in Hungary: results of an epidemiological cross-sectional study
}

\author{
Péter Torzsa', Raghavendra Devadiga ${ }^{2}$ and Monica Tafalla ${ }^{3^{*}}$ (D)
}

\begin{abstract}
Background: Pertussis (whooping cough) is well known to be underreported, particularly among adults, who can act as an infectious reservoir, potentially putting susceptible newborns at risk of serious illness. The purpose of this study was to estimate the seroprevalence of pertussis in adults in Hungary.

Methods: This epidemiological, cross-sectional study was conducted in adults in five general practitioners' practices in Hungary. Serum anti-pertussis toxin immunoglobulin G (anti-PT lgG) antibody levels were analyzed using enzyme-linked immunosorbent assay. Sera were classified following manufacturer's instructions as: strongly indicative of current/recent infection ( $\geq 1.5$ optical density [OD] units); indicative of current/recent infection ( $\geq 1.0$ OD units); seropositive ( $>0.3$ OD units); or seronegative ( $\leq 0.3 \mathrm{OD}$ units). Logistic regression was performed to describe the associations between seroprevalence and various characteristics.

Results: Between 24 ${ }^{\text {th }}$ April 2014 and $24^{\text {th }}$ April 2015, 1999 adults (60.6\% female; mean age $47.4 \pm 17.7$ years) were included in the analysis. A total of $14.8 \%$ were seropositive for anti-PT IgG, 1.1\% had a level indicative of current/recent infection, and $0.1 \%$ had a level strongly indicative of current/recent infection. Logistic regression showed significant relationships between increased rates of seropositivity and: age $\geq 60$ years (odds ratio [OR], 1.97; 95\% confidence interval $[\mathrm{Cl}], 1.39-2.80 ; p=.0002)$ or $18-29$ years $(\mathrm{OR}, 1.67 ; 95 \% \mathrm{Cl}, 1.13-2.46 ; p=.0094)$ vs. 45-59 years; former smoker (OR, 1.46; $95 \% \mathrm{Cl}, 1.08-1.97 ; p=.014)$ or current smoker (OR, 1.38; 95\% Cl, 1.01-1.89; $p=.045)$ vs. never smoker; and male (OR, 1.30; $95 \% \mathrm{Cl}, 1.01-1.68 ; p=.041)$ vs. female. Also, between increased rates of probable current/recent infection and current smoker (OR, 7.50; 95\% Cl, 2.32-24.31; $p=.0008$ ) or former smoker (OR, 4.07; 95\% Cl, 1.21-13.64; $p=.023$ ) vs. never smoker.

Conclusions: Approximately $85 \%$ of the adults studied were seronegative and therefore susceptible to pertussis infection. Approximately $1 \%$ had anti-PT IgG levels indicative of current/recent pertussis infection, which could potentially be transmitted to susceptible young infants. Vaccination of adults is a key way to indirectly protect infants.
\end{abstract}

Trial registration: Clinical Trials.gov NCT02014519. Prospectively registered 12 December 2013.

Keywords: Seroprevalence, Bordetella pertussis, Epidemiology, Hungary

\section{Background}

Pertussis (whooping cough) is an acute respiratory tract infection caused by Bordetella pertussis that is characterized by a chronic, severe cough [1]. As pertussis is a potentially life-threatening infection for newborns and unvaccinated infants [2], vaccination during infancy is recommended. In Hungary, pertussis immunization using a combined diphtheria-tetanus-whole cell pertussis

\footnotetext{
* Correspondence: monica.x.tafalla@gsk.com

${ }^{3}$ GSK, Avenue Fleming 20, Wavre 1300, Belgium

Full list of author information is available at the end of the article
}

vaccine - was introduced in 1953 [3]. This had a huge beneficial impact on pertussis incidence [3]. In 2006, this was switched to a combined diphtheria-tetanus-acellular pertussis vaccine [4]. The optimum immunization schedule for pertussis is unclear, so these vary by country; in Hungary, the current recommended immunization schedule includes acellular pertussis at ages 2, 3, and 4 months, with boosters at ages 1.5, 6, and 11 years [5]. Coverage of all doses in Hungary is very high (>99\%) [6].

However, adults are at risk of pertussis infection due to waning immunity following immunization or natural 
infection [7-10]. Although pertussis during adulthood is generally not serious, it can result in morbidity and more importantly - infected adults can pass on the infection to infants who have not been (fully) vaccinated [9]. Some European countries have therefore introduced various combinations of: adolescent, adult, or elderly boosters; a "cocooning strategy" (vaccination of household members in contact with a newborn); or vaccination of pregnant women (to provide the infant with placentally transmitted antibodies) $[11,12]$. Apart from the introduction of adolescent boosters, these strategies have not yet been recommended in Hungary [11]. Given that the notification rate in infants (age <1 year) in Hungary was 2.24/100,000 in 2014, roughly 12 -fold higher than in those aged $\geq 1$ year (approximately $0.18 / 100,000)$ [13], young infants are clearly still at risk of pertussis despite high pediatric population vaccination coverage.

Although overall pertussis notification rates in Hungary are among the lowest in Europe [13], at 0.17/ 100,000 in 2015 [14, 15], it is well known that pertussis is underreported, particularly among adults, who generally have much milder symptoms than infants, making it difficult to distinguish from a common cold [16]. The low notification rate of pertussis coupled with the high immunization coverage may result in general practitioners (GPs) in Hungary not testing adults with chronic cough for pertussis. The true incidence of pertussis may therefore be considerably higher than the notified cases, particularly among adults.

A more realistic estimate of pertussis incidence can be obtained from seroepidemiological studies; which can also help in the determination of immunity duration, the need for booster doses, and to investigate disease resurgence and its causes [17]. However, although high antibody titers are indicative of recent infection, lower levels can be due to more distant infection or vaccination. Although higher anti-pertussis toxin immunoglobulin G (anti-PT IgG) levels have been correlated with protection against pertussis [18], there is no agreement on the level of pertussis antibodies that confers protection against pertussis. This makes the interpretation of antibody prevalence data challenging. A number of studies have examined the seroprevalence of pertussis antigens in various countries, but as no such studies have yet been undertaken in Hungary, the purpose of this seroepidemiological study was to estimate the seroprevalence of pertussis in adults in Hungary.

\section{Methods}

This epidemiological, cross-sectional study (NCT02014519) was conducted in adults in five centers in Hungary. Subjects were recruited from GP clinics between $24^{\text {th }}$ April 2014 and $24^{\text {th }}$ April 2015, mostly during a clinic visit for another purpose, but some by telephone or email contact (particularly in the youngest age group, due to difficulties in recruitment). Subjects had to be aged $\geq 18$ years and provide written informed consent; and not have been vaccinated against pertussis within the previous 12 months or have a confirmed or suspected immunological disorder.

Serum anti-PT IgG levels were determined using a commercial enzyme-linked immunosorbent assay (ELISA) (PERTUSSCAN PT IgG, Euro Diagnostica AB, Sweden) at the Laboratory of the National Epidemiology Centre using standardized and validated procedures in accordance with the manufacturer's instructions [19]. Test results were used to classify sera according to manufacturer pre-defined cut-off values as: strongly indicative of current/recent infection ( $\geq 1.5$ optical density [OD] units; corresponds to 110 ELISA units [EU]/mL against the United States (US) Food and Drug Administration standard Lot 3 [19]); indicative of current/recent infection $(\geq 1.0 \mathrm{OD}$ units $[70 \mathrm{EU} / \mathrm{mL}])$; seropositive $(>0.3$ OD units $[18 \mathrm{EU} / \mathrm{mL}]$; the sensitivity limit of the assay); or seronegative ( $\leq 0.3$ OD units).

The main aim of the study was to assess the seroprevalence of anti-PT IgG in adults in Hungary, according to the cut-off levels defined above; this was also assessed according to age category $(18-29,30-44,45-59$, and $\geq 60$ years). Comparisons of seropositivity and anti-PT IgG levels indicative of current/recent infection by age category were tested using the Chi-square test. Relationships between seropositivity and age category, gender, history of vaccination against pertussis, history of pertussis infection, medication (antibiotics related to respiratory infections, prescribed and over-the-counter cough medicines) in the previous 12 months, hospitalization due to respiratory infections in the previous 12 months, and smoking status were examined by a logistic regression analysis (using the method of maximum likelihood to estimate the parameters). The same was done for antibody levels indicative of current/recent infection. In addition to the saturated models (i.e., considering all of the potential factors), final models using the backward elimination strategy (in which a $p$-value of 0.20 is required to keep a potential factor in the model) were also performed. Both models adjusted for all other factors in the model, including confounders. A $p$-value <.05 was taken to be statistically significant. All statistical analyses were performed using SAS Version 9.2.

The target sample size was approximately 2000 subjects (400 aged 18-29 years, 550 aged $30-44$ years, 510 aged 45-59 years, 540 aged $\geq 60$ years), based on a previous similar study in Israel [20].

\section{Results}

A total of 1999 subjects valid for analysis were enrolled (one subject was excluded for violation of inclusion and exclusion criteria). Demographics are shown in Table 1. It should be noted that 836 subjects did not know their 
Table 1 Characteristics of the study population, overall and by seropositivity for anti-PT IgG

\begin{tabular}{|c|c|c|c|}
\hline & Total $(n=1999)$ & Seropositive $^{\mathrm{a}}(n=295)$ & Seronegative $^{\mathrm{b}}(n=1704)$ \\
\hline \multicolumn{4}{|l|}{ Demographics } \\
\hline Age (years) & $47.4 \pm 17.7$ & $48.8 \pm 19.4$ & $47.1 \pm 17.4$ \\
\hline Female & 1212/1999 (60.6) & 160/295 (54.2) & 1052/1704 (61.7) \\
\hline \multicolumn{4}{|l|}{ Pertussis-related history } \\
\hline Pertussis vaccination & $882 / 1163(75.8)$ & $127 / 165(77.0)$ & 755/998 (75.7) \\
\hline Prior pertussis & 88/1566 (5.6) & 15/227 (6.6) & 73/1339 (5.5) \\
\hline Infant & 3/87 (3.4) & 0 & 3/73 (4.1) \\
\hline Child & 75/87 (86.2) & 13/14 (92.9) & 62/73 (84.9) \\
\hline Adolescent & 3/87 (3.4) & $1 / 14(7.1)$ & $2 / 73(2.7)$ \\
\hline Adult & 6/87 (6.9) & 0 & $6 / 73(8.2)$ \\
\hline Long-lasting cough ${ }^{c}$ & 164/1971 (8.3) & 20/290 (6.9) & 144/1681 (8.6) \\
\hline $\begin{array}{l}\text { Recent contact with a person with } \\
\text { pertussis/long-lasting cough }{ }^{c}\end{array}$ & 227/1795 (12.6) & $30 / 270(11.1)$ & 197/1525 (12.9) \\
\hline Regular contact with children & 919/1999 (46.0) & $122 / 295(41.4)$ & 797/1704 (46.8) \\
\hline Relevant medications $^{\mathrm{d}}$ & 241/1962 (12.3) & 39/287 (13.6) & 202/1675 (12.1) \\
\hline Relevant hospitalizations ${ }^{\mathrm{e}}$ & 10/1994 (0.5) & 4/294 (1.4) & $6 / 1700(0.4)$ \\
\hline
\end{tabular}

Data are mean $\pm S D$ or $n / N(\%)$, where $N$ is the number of subjects with known data

Abbreviations: Anti-PT IgG anti-pertussis toxin immunoglobulin G, OD optical density, SD standard deviation

${ }^{\mathrm{a}}>0.3 \mathrm{OD}$ units

$\mathrm{b}^{\mathrm{a}} \leq 0.3$ OD units

CLasting $\geq 3$ weeks in the previous 12 months

${ }^{\mathrm{d}}$ Any antibiotics and/or other medication (i.e., any cough medicines) for lower respiratory tract infections or (suspected) pertussis infections in the previous 12 months

eHospitalized due to respiratory infections in the previous 12 months

vaccination status. Of those who knew whether they had received a pertussis vaccination, $75.8 \%$ had been vaccinated. Only $5.6 \%$ of subjects who knew their pertussis history reported having been previously diagnosed with pertussis, most often as a child (86.2\%). Among those who were seropositive, none reported having been previously diagnosed with pertussis as an adult. Among those who reported a long-lasting cough in the last 12 months (8.3\%), most (86.4\%) reported that their cough had lasted for $\geq 30$ days and were dry (54.3\%). Contact with a person with a long-lasting cough in the previous 12 months was reported by $12.6 \%$ of subjects, mainly with a family member $(61.7 \%)$ and daily contact (67.8\%). Only $0.5 \%$ of the subjects had been hospitalized due to respiratory infections in the previous 12 months, a median (range) of 1 (1-5) times, for a median (range) of 9 (3-35) days.

A total of 295 subjects (14.8\%) were seropositive for anti-PT IgG (>0.3 OD units), $22(1.1 \%)$ had a level indicative of current/recent infection ( $\geq 1.0$ OD units), and $2(0.1 \%)$ had a level strongly indicative of current/recent infection ( $\geq 1.5$ OD units) (Table 2). Seropositivity $(>0.3$ OD units) varied significantly across age groups $(p=.0004)$, and was the highest among those aged $\geq 60$ years and 18-29 years; but current/recent pertussis infection status $(\geq 1.0$ OD) did not vary significantly by age $(p=.096)$.
Various characteristics were significantly associated with seropositivity ( $>0.3$ vs. $\leq 0.3$ OD units), namely age (age 18-29 and $\geq 60$ vs. $45-59$ years), gender, and smoking status (Table 3). For increased likelihood of current/recent infection ( $\geq 1.0$ vs. $<1.0$ OD units), only smoking status remained significant after backward elimination; although there was a trend towards increased likelihood of current/recent infection among those aged $\geq 60$ years (Table 4 ).

\section{Discussion}

In this Hungarian study, $14.8 \%$ of the healthy adults tested (during 2014-2015) were seropositive ( $>0.3$ OD units) for anti-PT IgG. This is lower than has been reported in seroprevalence studies in adults in other countries (approximately 20-80\% [20-27]). However, it should be noted that these studies used a variety of different assays; and the units and cut-offs used to define seropositivity varied widely, which limits their comparability [16]. Further, seropositivity for anti-PT IgG can be due to prior pertussis infection or vaccination [28]. Although none of the subjects in the current study had been vaccinated against pertussis within the last year, this was not necessarily the case for the other seroepidemiological studies. Regardless of these issues, in the current study, $85.2 \%$ of the adults tested were seronegative and therefore at risk of 


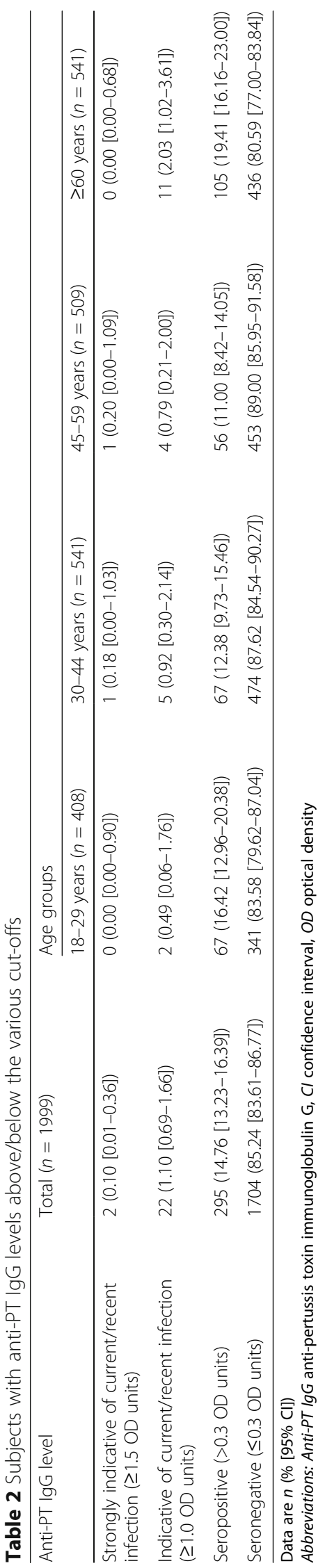


Table 3 Estimated coefficients of the fitted logistic regression model for seropositivity, ${ }^{a}$ before and after backward elimination

\begin{tabular}{|c|c|c|c|c|}
\hline \multirow[t]{2}{*}{ Characteristics } & \multicolumn{2}{|l|}{ Saturated model } & \multicolumn{2}{|l|}{ Final model } \\
\hline & OR $(95 \% \mathrm{Cl})$ & $p$-value & OR $(95 \% \mathrm{Cl})$ & $p$-value \\
\hline Age $18-29$ vs. $45-59$ years $^{b}$ & $1.64(1.10-2.44)$ & .015 & $1.67(1.13-2.46)$ & .0094 \\
\hline Age $30-44$ vs. $45-59$ years $^{\mathrm{b}}$ & $1.15(0.78-1.70)$ & .48 & $1.17(0.80-1.71)$ & .41 \\
\hline Age $\geq 60$ vs. $45-59$ years $^{b}$ & $2.13(1.46-3.12)$ & $<.0001$ & $1.97(1.39-2.80)$ & .0002 \\
\hline Male vs. female & $1.29(1.00-1.67)$ & .050 & $1.30(1.01-1.68)$ & .041 \\
\hline Vaccination yes vs. no & $1.46(0.92-2.29)$ & .11 & - & - \\
\hline Vaccination unknown vs. no & $1.36(0.90-2.05)$ & .15 & - & - \\
\hline Pertussis yes vs. no & $0.99(0.54-1.81)$ & .97 & - & - \\
\hline Pertussis unknown vs. no & $0.99(0.72-1.37)$ & .96 & - & - \\
\hline Medication ${ }^{c}$ yes vs. no & $1.04(0.71-1.54)$ & .83 & - & - \\
\hline Medication ${ }^{c}$ unknown vs. no & $1.44(0.62-3.33)$ & .39 & - & - \\
\hline Hospitalization ${ }^{d}$ yes vs. no & $3.01(0.79-11.55)$ & .11 & - & - \\
\hline Hospitalization ${ }^{d}$ unknown vs. no & $1.03(0.11-10.21)$ & .98 & - & - \\
\hline Current vs. never smoker & $1.36(0.99-1.87)$ & .055 & $1.38(1.01-1.89)$ & .045 \\
\hline Former vs. never smoker & $1.44(1.07-1.95)$ & .018 & $1.46(1.08-1.97)$ & .014 \\
\hline
\end{tabular}

Abbreviations: $C l$ confidence interval, $O D$ optical density, $O R$ odds ratio, vs. versus

${ }^{\mathrm{a}}>0.3$ OD units

${ }^{\mathrm{b}}$ The age group with the lowest seropositivity was used as the reference

${ }^{c}$ Any antibiotics and/or other medication (i.e., any cough medicines) for lower respiratory tract infections or (suspected) pertussis infections in the previous 12 months

${ }^{\mathrm{d}}$ Hospitalized due to respiratory infections in the previous 12 months

Table 4 Estimated coefficients of the fitted logistic regression model for increased likelihood of current/recent infection, ${ }^{\mathrm{b}}$ before and after backward elimination

\begin{tabular}{|c|c|c|c|c|}
\hline \multirow[t]{2}{*}{ Characteristics } & \multicolumn{2}{|l|}{ Saturated model } & \multicolumn{2}{|l|}{ Final model } \\
\hline & OR $(95 \% \mathrm{Cl})$ & $p$-value & OR $(95 \% \mathrm{Cl})$ & $p$-value \\
\hline Age $18-29$ vs. $45-59$ years $^{b}$ & $0.57(0.10-3.31)$ & .53 & $0.58(0.11-3.24)$ & .54 \\
\hline Age $30-44$ vs. $45-59$ years $^{b}$ & $1.21(0.31-4.74)$ & .79 & $1.21(0.32-4.57)$ & .78 \\
\hline Age $\geq 60$ vs. $45-59$ years $^{\mathrm{b}}$ & $3.60(1.06-12.24)$ & .040 & $2.99(0.94-9.56)$ & .064 \\
\hline Male vs. female & $1.55(0.65-3.70)$ & .32 & - & - \\
\hline Vaccination yes vs. no & $2.03(0.35-11.81)$ & .43 & - & - \\
\hline Vaccination unknown vs. no & $3.31(0.71-15.5)$ & .13 & - & - \\
\hline Pertussis yes vs. no & $0.45(0.06-3.64)$ & .45 & - & - \\
\hline Pertussis unknown vs. no & $0.30(0.08-1.08)$ & .065 & - & - \\
\hline Medication ${ }^{c}$ yes vs. no & $1.24(0.35-4.38)$ & .74 & - & - \\
\hline Medication ${ }^{c}$ unknown vs. no & $2.14(0.25-17.97)$ & .49 & - & - \\
\hline Hospitalization ${ }^{d}$ yes vs. no & $0.00(0.00-N R)$ & .99 & - & - \\
\hline Hospitalization ${ }^{d}$ unknown vs. no & $0.00(0.00-\mathrm{NR})$ & 1.00 & - & - \\
\hline Current vs. never smoker & $7.08(2.17-23.16)$ & .0012 & $7.50(2.32-24.31)$ & .0008 \\
\hline Former vs. never smoker & $4.12(1.21-14.01)$ & .024 & $4.07(1.21-13.64)$ & .023 \\
\hline
\end{tabular}

Abbreviations: $C l$ confidence interval, $N R$ not reported, $O D$ optical density, $O R$ odds ratio, vs. versus

$\mathrm{a}_{\geq 1.0}$ OD units

${ }^{\mathrm{b}}$ The age group with the lowest seropositivity was used as the reference

'Any antibiotics and/or other medication (i.e., any cough medicines) for lower respiratory tract infections or (suspected) pertussis infections in the previous 12 months

${ }^{d}$ Hospitalized due to respiratory infections in the previous 12 months 
pertussis infection. Although childhood vaccination rates in Hungary are very high [6], immunity to pertussis wanes over time [7-10], meaning that many adults are no longer protected. This could be overcome by the introduction of adult pertussis boosters, an approach that has already been adopted in various European countries [11] and the US [29].

In the current study, $1.1 \%$ of subjects had anti-PT IgG levels $\geq 1.0$ OD (indicative of current/recent infection). It is difficult to compare this with other studies, which have used a range of different assays, cut-offs, and definitions, but infection within the last year/recent/active infection rates of approximately 1-10\% have been reported in adults [22, 25, 27, 30-36], which are similar to or higher than in the current study.

In order to try to compare our seroprevalence results with notified cases of pertussis, an estimation of pertussis incidence based on seroprevalence data combined with the manufacturer pre-defined cut-off values was undertaken. Previously, de Melker et al. [37] used seroprevalence data combined with antibody decline over time after infection to estimate incidence. Assuming that "recent" relates to the previous year, 2/1999 subjects $(0.1 \%)$ with a level strongly indicative of current/recent infection ( $\geq 1.5$ OD units) in the current study would translate into a crude annual incidence of pertussis infection of $100 / 100,000$. Similarly, the 22/1999 subjects (1.1\%) with a level indicative of current/recent infection ( $\geq 1.0$ OD units) would give a crude annual incidence of $1100 / 100,000$. These crude incidences are roughly 500and 6000-fold higher than the notified rate among those aged $\geq 20$ years in Hungary in 2014 (approximately 0.19 / 100,000) [13], indicating a very high level of underreporting. A recent review has found similar discrepancies all around the world [16]. However, it should be noted that the current study was not designed to perform this type of calculation and "recent" (as defined by the manufacturer) may not equate to 1 year. Therefore, these estimations and comparisons should be interpreted with caution.

The introduction of adult pertussis boosters could help alleviate the burden of pertussis in this age group. It may also help reduce the risk of transmission to susceptible infants. Other approaches to protect infants prior to their primary immunizations include cocooning or immunization of pregnant women. Various European countries [11] and the US [29] have introduced one or other of these approaches to help protect newborns. However, recent data indicate that post-partum vaccination of parents may not be as successful as maternal vaccination during pregnancy [38-40]. Also, although maternal immunization may benefit newborns, this approach alone would not address the burden of pertussis in the adult population.
In the current study, seropositivity was significantly more likely among those aged $\geq 60$ and $18-29$ years vs. 45-59 years; and there was a trend towards more likely current/recent infection among those aged $\geq 60$ years vs. 45-59 years. Overall, these results support waning immunity following childhood vaccinations, ultimately resulting in higher levels of infection during later years. The pertussis booster at age 11 years was introduced in Hungary in 2006 [4], so some of the adults in the youngest age group could have received this vaccination approximately 7 or 8 years earlier. The remainder of the population would probably have last received pertussis vaccination $\geq 14$ years ago; or never have been vaccinated. Although immunity wanes quite quickly after pertussis vaccination [41], anti-PT antibodies can persist for up to approximately 10 years [42], therefore the elevated rate of seropositivity in the 18-29-year age group is likely due to adolescent (or even childhood) vaccination. Among those aged $\geq 60$ years, the elevated seropositivity is likely related to pertussis infection, which was also elevated in this age group. Based on these results, vaccination of the elderly could be beneficial in Hungary to address the pertussis burden in this age group, who can experience considerable pertussis-related morbidity [43].

Some other studies have also reported higher seropositivity among older adults [20, 23, 27]. However, others have reported highest seropositivity rates in various younger adult age groups [21, 24] or little variation by age among adults [25]. Similarly, some previous studies have reported a possible link between likely pertussis infection and advancing age [20, 22, 31], but others have reported no such link $[25,34,35]$. Whether these results are indicative of true differences between countries and/ or years or whether they are due to a lack of sufficient power to detect such differences is unclear.

Regarding gender, we found that males were significantly more likely to be seropositive than females; but not significantly more likely to have anti-PT IgG levels indicative of current/recent infection. Higher male seropositivity has also been reported in studies from Mexico ( $p=.0007)$ [24] and Korea $(p=.023)$ [23]; but studies from Spain [26], Greece [21], and The Gambia [22] have reported no significant differences in seropositivity between genders. Regarding higher male likelihood of recent infection, this has been reported to be significant in a study from The Netherlands [31], non-significant in a study from Denmark [34], higher but significance not reported in one from The Gambia [22], or no significant gender differences in two studies from China $[35,36]$. Overall, it is unclear whether there is a true link between pertussis and gender and, if there is, the cause is unknown.

Regarding smoking status, we found correlations between seropositivity and current/former smoking, and 
even stronger correlations between evidence of recent pertussis infection and current/former smoking. This could be because the epithelial cells of smokers have been shown to have enhanced binding to Bordetella pertussis [44], thus increasing pertussis infection risk. However, a seroepidemiology study in Denmark found no significant link between smoking status and recent pertussis infection [34].

\section{Limitations}

Some of the subjects were enrolled into this study at a regular GP visit, so the studied population may be sicker and/or more health-conscious than the general population. This method of recruitment probably also accounts for the higher proportion of female subjects. Unfortunately, data on the route of recruitment (GP visit, telephone call or email) were not collected, so it is not possible to compare results by route of recruitment. Also, although we recognize that this "convenience sample" is a limitation of this study, we feel that it is likely representative of the general Hungarian adult population.

As there is no agreed threshold of anti-PT antibodies that provide protection, seroprevalence studies are notoriously difficult to interpret. Also, the manufacturer's definition of "current/recent" infection is rather vague, making comparisons with other studies difficult. Lastly, $p$-values were not adjusted for multiplicity of endpoints (as there was only one endpoint of interest), so the statistical significance of the results should be interpreted with caution.

\section{Conclusions}

In this seroprevalence study, $14.8 \%$ of adults in Hungary were found to be seropositive for anti-PT IgG antibodies, with increased risk among males, those aged $\geq 60$ or 18 29 years, and current/former smokers. The remaining $85.2 \%$ of adults were seronegative, and therefore at risk of pertussis infection. Furthermore, $1.1 \%$ of adults had evidence of current/recent pertussis infection, with increased risk among current/former smokers and a trend towards an increased risk among those aged $\geq 60$ years. This gives an estimated crude annual pertussis incidence of 1100 / 100,000 , roughly 6000 -fold higher than the notified rate in Hungary. This indicates that many adults in Hungary may have undiagnosed pertussis, and this could be unknowingly transmitted to susceptible infants. The introduction of additional pertussis boosters during adulthood could help overcome this potential issue.

\section{Abbreviations}

Anti-PT IgG: Anti-pertussis toxin immunoglobulin G; Cl: Confidence interval; ELISA: Enzyme-linked immunosorbent assay; EU: ELISA units; GP: General practitioner; OD: Optical density; SD: Standard deviation; US: United States

\section{Acknowledgements}

The authors thank Dr. Katalin Faragó (principal investigator) for her contribution to the study, Amulya Jayadev (statistician, GSK) for statistical support and Andrew Vyse (epidemiologist, GSK) for his valuable input provided during the review of the manuscript.

The authors would like to thank Business \& Decision Life Sciences platform for editorial assistance and manuscript coordination, on behalf of GSK.

Grégory Leroux coordinated manuscript development and editorial support.

The authors also thank Jenny Lloyd (Compass Healthcare Communications

Ltd., on behalf of GSK) for providing medical writing support.

\section{Funding}

GlaxoSmithKline Biologicals SA funded this study (NCT02014519) and was involved in all stages of study conduct, including analysis of the data. GlaxoSmithKline Biologicals SA also took in charge all costs associated with the development and publication of this manuscript.

\section{Availability of data and materials \\ The results summary for this study (GSK study number 116804 - NCT02014519) is currently available on the GSK Clinical Study Register and can be accessed at www.gsk-clinicalstudyregister.com/study/116804\#rs. For interventional studies that evaluate our medicines, anonymized patient-level data will be made available to independent researchers, subject to review by an independent panel, at www.clinicalstudydatarequest.com within 6 months of publication. To protect the privacy of patients and individuals involved in our studies, GSK does not publicly disclose subject-level data.}

\section{Authors' contributions}

$\mathrm{RD}$ and MT contributed to the design of the study, and analyzed and interpreted study data; PT and MT collected or generated the data. All authors participated in the development of this manuscript and in its critical review with important intellectual contributions. All authors had full access to the data and gave final approval before submission. All authors agree to be accountable for all aspects of the work in ensuring that questions related to the accuracy or integrity of any part of the work are appropriately investigated and resolved. The work described was carried out in accordance to ICMJE recommendations for conduct, reporting, editing, and publications of scholarly work in medical journals. The corresponding author had final responsibility to submit for publication.

\section{Competing interests}

PT declares that he has no competing interests. RD and MT are employees of the GSK group of companies. MT holds shares in the GSK group of companies as part of her employee remuneration.

\section{Consent for publication}

Not applicable.

\section{Ethics approval and consent to participate}

The study protocol, any amendments, the informed consent, and other information that required pre-approval were reviewed and approved by the Central Ethics Committee of the Medical Research Council - ETT-TUKEB. The study was conducted in accordance with all applicable regulatory requirements and the International Conference on Harmonisation (ICH) Guideline for Good Clinical Practice, all applicable subject privacy requirements, and the guiding principles of the Declaration of Helsinki. Written informed consent was obtained from each subject prior to the performance of any study-specific procedures.

\section{Publisher's Note}

Springer Nature remains neutral with regard to jurisdictional claims in published maps and institutional affiliations.

\section{Author details}

${ }^{1}$ Faculty of Medicine, Department of Family Medicine, Semmelweis University, Kútvölgyi út 4, Budapest 1125, Hungary. ${ }^{2}$ GSK, CDOC-B, \#5 Embassy Links, S.R.T. Road, Bangalore 560052, India. ${ }^{3}$ GSK, Avenue Fleming 20, Wavre 1300, Belgium. 


\section{Received: 15 October 2016 Accepted: 28 March 2017}

\section{Published online: 04 April 2017}

\section{References}

1. Pertussis. European Centre for Disease Prevention and Control (ECDC). 2016. http://ecdc.europa.eu/en/healthtopics/pertussis/Pages/index.aspx. Accessed 22 Jun 2016.

2. Torre JA, Benevides GN, de Melo AM, Ferreira CR. Pertussis: the resurgence of a public health threat. Autops Case Rep. 2015;5:9-16.

3. Joo I. Epidemiology of pertussis in Hungary. Dev Biol Stand. 1991;73:357-9.

4. Heininger U, Andre P, Chlibek R, Kristufkova Z, Kutsar K, Mangarov A, et al. Comparative epidemiologic characteristics of Pertussis in 10 central and eastern European countries, 2000-2013. PLoS One. 2016;11:e0155949.

5. Vaccine Schedule. Immunisation schedules by target disease [pertussis] in [Hungary]. European Centre for Disease Prevention and Control (ECDC). 2016. http://vaccine-schedule.ecdc.europa.eu/Pages/Scheduler.aspx. Accessed 20 Jun 2016.

6. Országos Epidemiológiai Központ [National Epidemiology Center]. Védőoltások teljesítése, 2015 [Vaccinations performance of 2015]. Epinfo. 2016;23:369-81. http://www.oek.hu/oekfile.pl?fid=6840. Accessed 8 Sep 2016.

7. Esposito S, Principi N, European Society of Clinical Microbiology and Infectious Diseases (ESCMID) Vaccine Study Group (EVASG). Immunization against pertussis in adolescents and adults. Clin Microbiol Infect. 2016 22(Suppl 5):S89-S95.

8. Wendelboe AM, Van Rie A, Salmaso S, Englund JA. Duration of immunity against pertussis after natural infection or vaccination. Pediatr Infect Dis J. 2005;24(5 Suppl):S58-61.

9. Zepp F, Heininger U, Mertsola J, Bernatowska E, Guiso N, Roord J, et al. Rationale for pertussis booster vaccination throughout life in Europe. Lancet Infect Dis. 2011:11:557-70.

10. World Health Organization (WHO). Pertussis vaccines: WHO position paper, August 2015 - Recommendations. Vaccine. 2016;34:1423-5.

11. Recommended immunisations for pertussis [All EU countries]. European Centre for Disease Prevention and Control (ECDC). 2016. http://vaccine-schedule.ecdc. europa.eu/Pages/Scheduler.aspx. Accessed 19 Jul 2016.

12. European Centre for Disease Prevention and Control (ECDC). Expert consultation on pertussis 2012. http://ecdc.europa.eu/en/publications/ Publications/pertussis-meeting-2012.pdf. Accessed 22 Jun 2016.

13. Surveillance Atlas of Infectious Diseases. European Centre for Disease Prevention and Control (ECDC). 2016. http://atlas.ecdc.europa.eu/public/index. aspx?. Accessed 22 June 2016.

14. Országos Epidemiológiai Központ [National Epidemiology Center]. Fertőző betegségek adatai [data on infectious diseases]. Epinfo. 2016;22:640-50. http://www.oek.hu/oekfile.pl?fid=6451. Accessed 5 Sept 2016.

15. Population Pyramids of the World from 1950 to 2100 . Hungary 2015. http://populationpyramid.net/hungary/2015/. Accessed 8 Sep 2016

16. Barkoff AM, Gröndahl-Yli-Hannuksela K, He Q. Seroprevalence studies of pertussis: what have we learned from different immunized populations. Pathog Dis. 2015;73:ftv050.

17. Cutts FT, Hanson M. Seroepidemiology: an underused tool for designing and monitoring vaccination programmes in low- and middle-income countries. Tropical Med Int Health. 2016;21:1086-98.

18. Taranger J, Trollfors B, Lagergård T, Sundh V, Bryla DA, Schneerson R, et al. Correlation between pertussis toxin $\lg G$ antibodies in postvaccination sera and subsequent protection against pertussis. J Infect Dis. 2000;181:1010-3.

19. Euro Diagnostica. Instruction: PERTUSSCAN PT IgG: ELISA for detection of lgG against Bordatella pertussis toxin (PT) in serum. Malmö: Euro Diagnostica; 2013. p. 120. Document No.: E-23-0207-02

20. Rendi-Wagner P, Tobias J, Moerman L, Goren S, Bassal R, Green M, et al. The seroepidemiology of Bordetella pertussis in Israel - estimate of incidence of infection. Vaccine. 2010;28:3285-90.

21. Pavlopoulou ID, Syriopoulou V, Daikos GL, Fourlani H, Tzivaras A, Petychakis D, et al. Pertussis seroprevalence in different age groups in Greece. Scand J Infect Dis. 2007:39:14-8

22. Scott S, van der Sande M, Faye-Joof T, Mendy M, Sanneh B, Barry Jallow F, et al. Seroprevalence of pertussis in the Gambia: evidence for continued circulation of bordetella pertussis despite high vaccination rates. Pediatr Infect Dis J. 2015:34:333-8.

23. Lee SY, Han SB, Bae EY, Kim JH, Kang JH, Park YJ, et al. Pertussis seroprevalence in korean adolescents and adults using anti-pertussis toxin immunoglobulin G. J Korean Med Sci. 2014;29:652-6.
24. Conde-Glez C, Lazcano-Ponce E, Rojas R, DeAntonio R, Romano-Mazzotti L, Cervantes $Y$, et al. Seroprevalence of Bordetella pertussis in the Mexican population: a cross-sectional study. Epidemiol Infect. 2014;142:706-13.

25. Wanlapakorn N, Ngaovithunvong V, Thongmee T, Vichaiwattana P, Vongpunsawad S, Poovorawan Y. Seroprevalence of antibodies to Pertussis toxin among different age groups in Thailand after 37 years of universal whole-cell Pertussis vaccination. PLoS One. 2016;11:e0148338.

26. González-Escalada A, García-García L, Viguera-Ester P, Marín-García P, García J, Gil-de-Miguel A, et al. Seroprevalence of antibodies against measles, rubella, mumps, varicella-zoster, and B. Pertussis in young adults of Madrid, Spain. Hum Vaccin Immunother. 2013:9:1918-25.

27. Palazzo R, Carollo M, Fedele G, Rizzo C, Rota MC, Giammanco A, et al. Evidence of increased circulation of Bordetella pertussis in the Italian adult population from seroprevalence data (2012-2013). J Med Microbiol. 2016: [Epub ahead of print].

28. Wirsing von König $\mathrm{CH}$. Pertussis diagnostics: overview and impact of immunization. Expert Rev Vaccines. 2014;13:1167-74.

29. Recommended Adult Immunization Schedule for Adults Aged 19 Years or Older, by Vaccine and Age Group. United States, 2016. Centers for Disease Control and Prevention (CDC). 2016. http://www.cdc.gov/vaccines/ schedules/hcp/imz/adult.html. Accessed 20 Jul 2016.

30. Caboré RN, Piérard D, Huygen K. A Belgian Serosurveillance/Seroprevalence Study of Diphtheria, Tetanus and Pertussis Using a Luminex XMAP Technology-Based Pentaplex. Vaccines (Basel). 2016:4:E16.

31. de Greeff SC, de Melker HE, van Gageldonk PG, Schellekens JF, van der Klis FR, Mollema L, et al. Seroprevalence of pertussis in The Netherlands: evidence for increased circulation of Bordetella pertussis. PLoS One. 2010;5:e14183.

32. Huygen K, Rodeghiero C, Govaerts D, Leroux-Roels I, Melin P, Reynders M, et al. Bordetella pertussis seroprevalence in Belgian adults aged 20-39 years, 2012. Epidemiol Infect. 2014;142:724-8.

33. Launay O, Toneatti C, Bernede C, Njamkepo E, Petitprez K, Leblond A, et al. Antibodies to tetanus, diphtheria and pertussis among healthy adults vaccinated according to the French vaccination recommendations. Hum Vaccin. 2009;5:341-6.

34. Rønn PF, Dalby T, Simonsen J, Jørgensen CS, Linneberg A, Krogfelt KA. Seroepidemiology of pertussis in a cross-sectional study of an adult general population in Denmark. Epidemiol Infect. 2014;142:729-37.

35. $X u Y$, , Wang $L, X u J$, Wang $X$, Wei $C$, Luo $P$, et al. Seroprevalence of pertussis in China: need to improve vaccination strategies. Hum Vaccin Immunother. 2014:10:192-8.

36. Chen Z, Zhang J, Cao L, Zhang N, Zhu J, Ping G, et al. Seroprevalence of pertussis among adults in China where whole cell vaccines have been used for 50 years. J Infect. 2016;73:38-44.

37. de Melker HE, Versteegh FG, Schellekens JF, Teunis PF, Kretzschmar M. The incidence of Bordetella pertussis infections estimated in the population from a combination of serological surveys. J Infect. 2006;53:106-13.

38. Carcione D, Regan AK, Tracey L, Mak DB, Gibbs R, Dowse GK, et al. The impact of parental postpartum pertussis vaccination on infection in infants: a population-based study of cocooning in Western Australia. Vaccine. 2015;33:5654-61.

39. Healy CM, Rench MA, Wootton SH, Castagnini LA. Evaluation of the impact of a pertussis cocooning program on infant pertussis infection. Pediatr Infect Dis J. 2015:34:22-6.

40. Safadi MA. Control of pertussis in infants: time has finally come? Expert Rev Vaccines. 2015;14:781-3.

41. McGirr A, Fisman DN. Duration of pertussis immunity after DTaP immunization: a meta-analysis. Pediatrics. 2015;135:331-43.

42. Mertsola J, Van Der Meeren O, He Q, Linko-Parvinen A, Ramakrishnan G, Mannermaa $L$, et al. Decennial administration of a reduced antigen content diphtheria and tetanus toxoids and acellular partussis vaccine in young adults. Clin Infect Dis. 2010;51:656-62.

43. Ridda I, Yin JK, King C, Raina Maclntyre C, McIntyre P. The importance of pertussis in older adults: a growing case for reviewing vaccination strategy in the elderly. Vaccine. 2012;30:6745-52.

44. El Ahmer OR, Essery SD, Saadi AT, Raza MW, Ogilvie MM, Weir DM, et al. The effect of cigarette smoke on adherence of respiratory pathogens to buccal epithelial cells. FEMS Immunol Med Microbiol. 1999;23:27-36. 